\title{
DESENVOLVIMENTO DE APLICATIVO COM REALIDADE AUMENTADA PARA O ENSINO DE DESENHO TÉCNICO, ELEMENTOS DE MÁQUINAS E PROJETOS MECÂNICOS
}

Beatriz B. N. Costa - beatriz.bonafe@aluno.ufabc.edu.br

Prof. Dr. Wallace G. Ferreira - wallace.ferreira@ ufabc.edu.br

Universidade Federal do ABC, Centro de Engenharia, Modelagem e Ciências Sociais Aplicadas

Avenida dos Estados, 5001, Bairro Santa Terezinha

09210-580 - Santo André - São Paulo

Pamila A. Costa - pamila.andrade@ hotmail.com

Prof. Dr. William M. Maluf Filho - wmaluf@ fei.edu.br

Centro Universitário da FEI, Engenharia Mecânica

Av. Humberto de Alencar Castelo Branco, 3972-B, Assunção

09850-901 - São Bernardo do Campo - São Paulo

Resumo: A Realidade Aumentada (RA) é uma tecnologia interativa que, por meio de informações computadorizadas, introduz objetos ou imagens virtuais no mundo real. Tal tecnologia pode ser utilizada em aplicativos para smartphones e tablets, sem a necessidade de um computador com alto processamento. A RA é uma tecnologia adequada para ser aplicada no âmbito educacional, permitindo maior interação do usuário com o objeto de estudo, estimulando seu interesse pelo assunto abordado e o aprendizado. Este artigo apresenta o desenvolvimento de aplicativo compativel com diferentes sistemas operacionais (em mais diferentes versões) para smartphones, para auxiliar no ensino de desenho técnico, elementos de máquinas e projetos mecânicos. $O$ aplicativo poderá ser utilizado em diferentes momentos para o aprendizado: seja em uma pré-preparação para as aulas, durantes as aulas de forma complementar ou extra-classe, seja para revisar os conteúdos estudados ou para a realização de exercícios. O presente projeto é uma parceria acadêmica entre a Universidade Federal do ABC (UFABC) e o Centro Universitário FEI. Em cada instituição, dois projetos de Iniciação Científica foram aprovados e estão em fase de implementação e testes pelas alunas, com orientação dos professores.

Palavras-chave: Realidade Aumentada. Educação. Engenharia. Desenho Técnico. Projetos Mecânicos. 


\section{INTRODUÇÃO}

Embora semelhantes em alguns aspectos, a Realidade Aumentada (RA) e a Realidade Virtual (RV) têm suas particularidades. A RV proporciona ao usuário um ambiente que simula a realidade. Para que tenha níveis satisfatórios de realismo, é necessária alta capacidade de processamento do dispositivo em que a aplicação é executada. Cientificamente, pode ser definida como por Kinner e Siscouto (2007) apud Fialho (2018):

“A Realidade Virtual (RV) é uma 'interface avançada do usuário' para acessar aplicações executadas no computador, propiciando a visualização, movimentação e interação do usuário, em tempo real, em ambientes tridimensionais gerados por computador".

$\mathrm{Na}$ RA, há a interação do ambiente virtual com o real, de modo que o primeiro sobrepõe o segundo e adiciona informações que não poderiam ser obtidas apenas com a percepção do usuário. O ambiente virtual na RA é uma expansão do mundo virtual, diferentemente da RV, em que há a tentativa de substituição. Conforme Macy apud Fialho (2018):

"O aspecto mais inovador da realidade aumentada não é a Tecnologia: é o objeto. Em vez de substituir objetos físicos com um computador, criamos sistemas que permitem as pessoas interajam com o mundo real de maneiras naturais e, ao mesmo tempo, beneficiem-se de capacidades aprimoradas do computador. O futuro que imaginamos não é o estranho mundo em que estamos inseridos na realidade virtual. Em vez disso, vemos nosso mundo familiar, aprimorado em diversos campos, muitas vezes invisíveis".

Com a aquisição de dispositivos móveis por meio da população após 2010, houve grande desenvolvimento e aplicação de RA com as mais diversas finalidades (MEKNI; LEMIEUX, 2014): entretenimento, publicidade, ciências humanas, biológicas e exatas, além da educação. Alguns exemplos podem ser verificados na Figura 1.

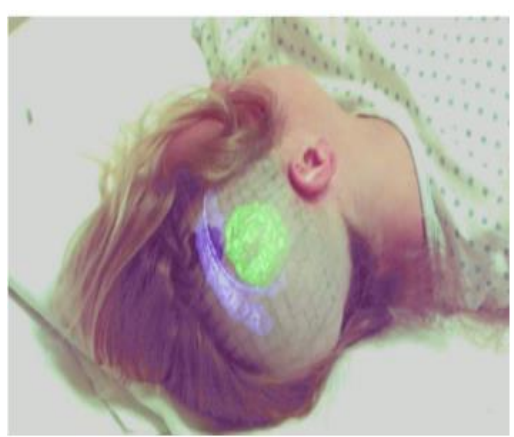

Figura 1 - Diferentes aplicações da RA.
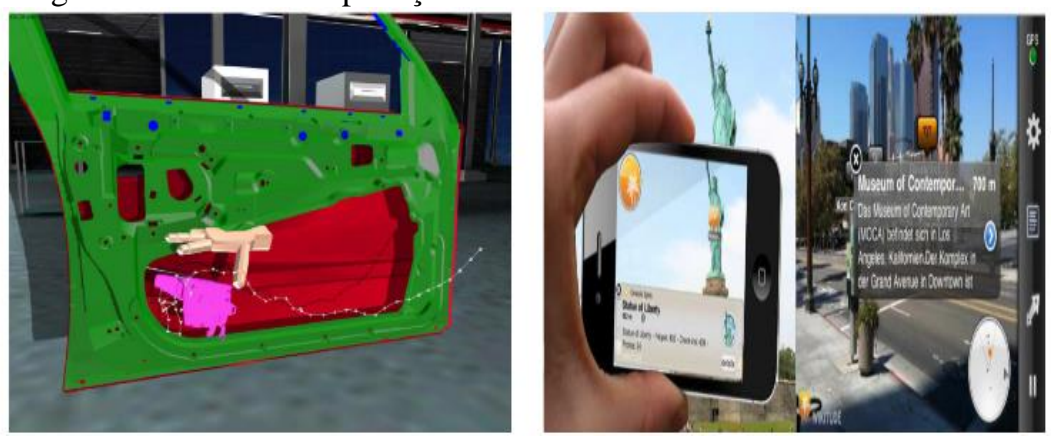

Fonte: Mekni; Lemieux (2014).

Para que possa haver a interação entre o mundo real e virtual, se faz necessária a utilização de dispositivos que transfiram as percepções sensoriais ao usuário. Em diversas aplicações são utilizados óculos, projetores, luvas, entre outros. Ao utilizar o smartphone ou o tablet, os componentes virtuais podem ser exibidos na própria tela do dispositivo, enquanto que sua câmera e sensores captam elementos do mundo real.

\section{OBJETIVOS E METODOLOGIA}

\subsection{Objetivos}

Ao longo do período do projeto, pretende-se complementar o conteúdo do livro Fundamentos de Desenho Técnico (RIASCOS et al., 2018) em uso nas aulas de desenho 
técnico nos cursos de engenharia da UFABC, sem que haja necessidade de adequações gráficas. O material impresso será exposto em sala de aula e complementado pelo aplicativo com seus recursos gráficos e informações via RA. No Centro Universitário FEI, por sua vez, existem listas de exercícios empregadas nas disciplinas de Elementos de Máquinas e Projetos Mecânicos que são usadas nas aulas do curso de Engenharia Mecânica. Estas listas também são complementadas com recursos de RA.

Com a necessidade de estar disponível para os usuários com diferentes perfis socioeconômicos, verifica-se que mais de $80 \%$ dos jovens entre 18 e 29 anos já possuíam celulares para uso pessoal em 2017, conforme censo (INSTITUTO BRASILEIRO DE GEOGRAFIA E ESTATÍSTICA, 2018). Assim, o desenvolvimento de um aplicativo educacional como aqui proposto é viável para a utilização no território brasileiro.

\subsection{Metodologia}

Para compreender quais aplicações de RA existem na educação de engenharia, foi realizada uma pesquisa na literatura técnica e científica, nacional e internacional. Assim, o objetivo é que o conteúdo proposto pelo aplicativo seja inovador e adequado com sua finalidade.

Foi necessário selecionar a plataforma de desenvolvimento e linguagem de programação. Para tal, houve pesquisa para que a escolha fosse adequada. A plataforma Unity (UNITY, [ $s$. d.]) foi selecionada para o desenvolvimento por sua relativa facilidade de programação, existência de componentes pré-programados, grande quantidade de recursos e a possibilidade de desenvolvimento para diferentes tipos de sistemas operacionais (como Android e iOS). Para a aplicação da RA, foi utilizada a plataforma Vuforia (PTC VUFORIA, [s. d.]).

Esses recursos são muito utilizados em videogames, aplicativos para celulares e ferramentas para diferentes propósitos, incluindo educacionais. Podem ser utilizados sem custo de licença ou royalties, desde que o aplicativo desenvolvido seja gratuito para utilização ou restrito a certos valores de faturamento sem fins lucrativos, como previstos em contrato.

\section{FUNDAMENTAÇÃO TEÓRICA}

\subsection{Revisão da literatura}

A definição de RA por diferentes autores está na Tabela 1.

Tabela 1 - Definição de RA por diferentes autores.

\begin{tabular}{|c|c|}
\hline Publicação & Definição da Tecnologia de RA \\
\hline $\begin{array}{l}\text { (CAUDELL; MIZELL, } \\
\text { 1992) }\end{array}$ & $\begin{array}{l}\text { Permite que usuários aumentem seu campo de visão através de } \\
\text { telas / displays }\end{array}$ \\
\hline (AZUMA, 1997) & $\begin{array}{c}\text { Permite aos usuários complementarem a realidade pela } \\
\text { sobreposição de objetos virtuais com os objetos de mundo real }\end{array}$ \\
\hline (AKÇAYIR et al., 2016) & $\begin{array}{c}\text { Sobrepõe a realidade virtual sobre os objetos do mundo real, em } \\
\text { todos os sentidos de percepção dos indivíduos. }\end{array}$ \\
\hline
\end{tabular}

Fonte: Autores.

Na última década, a utilização de RA em diferentes aplicações obteve resultados positivos em relação a seus objetivos, conforme diversos estudos (BILLINGHURST; CLARK; LEE, 2014; MARTIN et al., 2011; MEKNI; LEMIEUX, 2014; YIM; CHU; SAUER, 2017) Assim, organizações e desenvolvedores aperfeiçoam estes produtos e aplicativos. 
O artigo de revisão apresentado por Garzón, Pavón e Baldiris (2019), compila e discute a literatura técnica referente às aplicações da RA na área da educação. A maioria das aplicações de RA se concentra nos cursos das áreas de Ciências Naturais e Matemática, efetiva para o ensino de conceitos abstratos e complexos. O público alvo é, em geral, alunos de graduação ou bacharelado (ANTONIOLI; BLAKE; SPARKS, 2014; DIEGMANN et al., 2015; GARZÓN; ACEVEDO, 2019; IBÁÑEZ; DELGADO-KLOOS, 2018; OZDEMIR et al., 2018; RADU, 2014; SANTOS et al., 2014).

Na educação, adequa-se aos mais diferentes níveis de conhecimento. Na alfabetização até treinamentos técnicos. A RA é efetiva para o ensino de conceitos abstratos e complexos. Pode ser combinada com jogos educacionais, experimentos de laboratório, explicar um tópico, entre outras finalidades (BACCA et al., 2014). Exemplos de aplicação para o ensino de engenharia estão na Figura 2.

Figura 2 - Aplicações de RA em educação para engenharia.
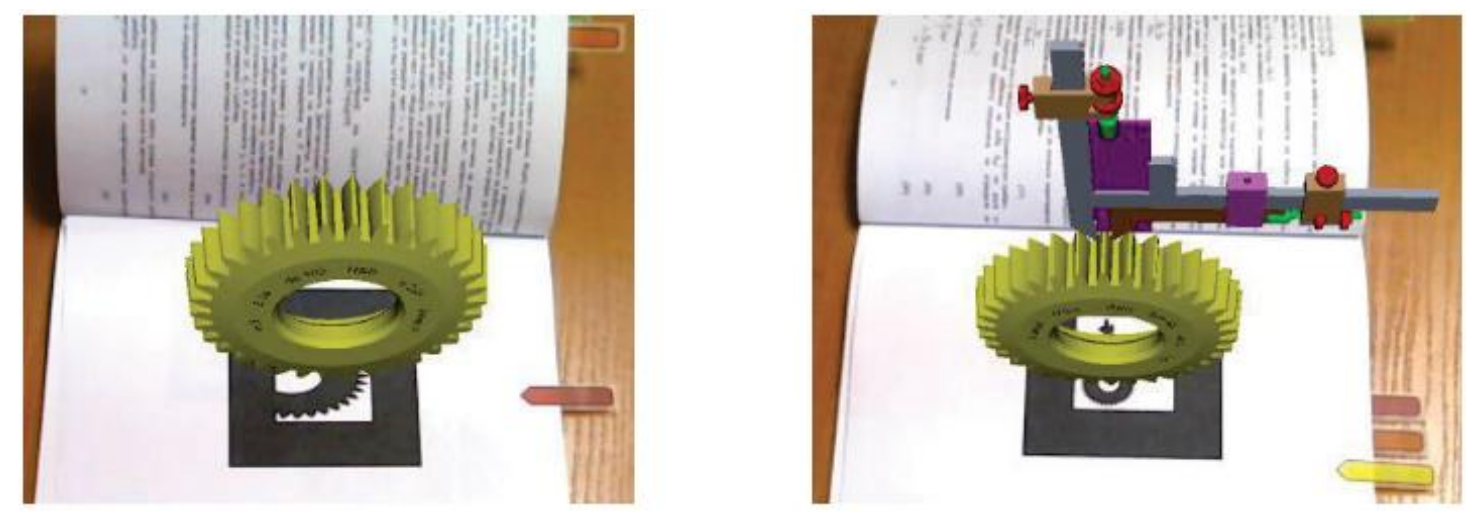

Fonte: Aliev et al. (2017).

O engajamento dos usuários com as aplicações e o auxílio delas para o aprendizado são fatos notáveis, conforme grande parte dos trabalhos supracitados. Há o auxílio do desenvolvimento da memória de longo prazo, especialmente na formação de estudantes de engenharia, artes e humanidades (GARZÓN; ACEVEDO, 2019). A RA permite que alunos e professores interajam em um ambiente de aprendizado colaborativo e ativo, estimulando a criatividade e a imaginação (BAZAROV et al., 2017).

\subsection{Princípios básicos da RA}

O princípio básico de funcionamento da RA é:

1) Um aplicativo desenvolvido e conectado a um repositório/plataforma na internet ou com dados previamente carregados, dispensando a conexão;

2) Sistema de câmeras ou diferentes sensores relacionados a um computador ou aparelho móvel (celular ou tablet), para que haja o processamento dos dados de entrada;

3) No que se tratam de sistemas que utilizam câmeras, é necessário um leitor de marcadores (targets) específicos, para ativar a realidade aumentada. A depender da tecnologia utilizada, pode se tratar de um código de barras, $Q R$ Code, ou até mesmo um desenho qualquer "codificado", desde que seja claramente identificado pelas câmeras.

4) Ao identificar o marcador, o aplicativo de RA insere o elemento virtual que foi associado ao marcador, podendo ser visualizado e manipulado no ambiente real. Em um aparelho móvel, pode haver interação do usuário com a tela sensível ao toque do próprio dispositivo, botões. 
(C) COBENGE 2020

"Os desafios para formar hoje o engenheiro do amanhã"
$\mathrm{Ol}$ a $\mathrm{O} 3$ de dezembro Evento On-line

O processo sobrescrito está apresentado de forma esquemática na Figura 3.

Figura 3 - Funcionamento da RA.

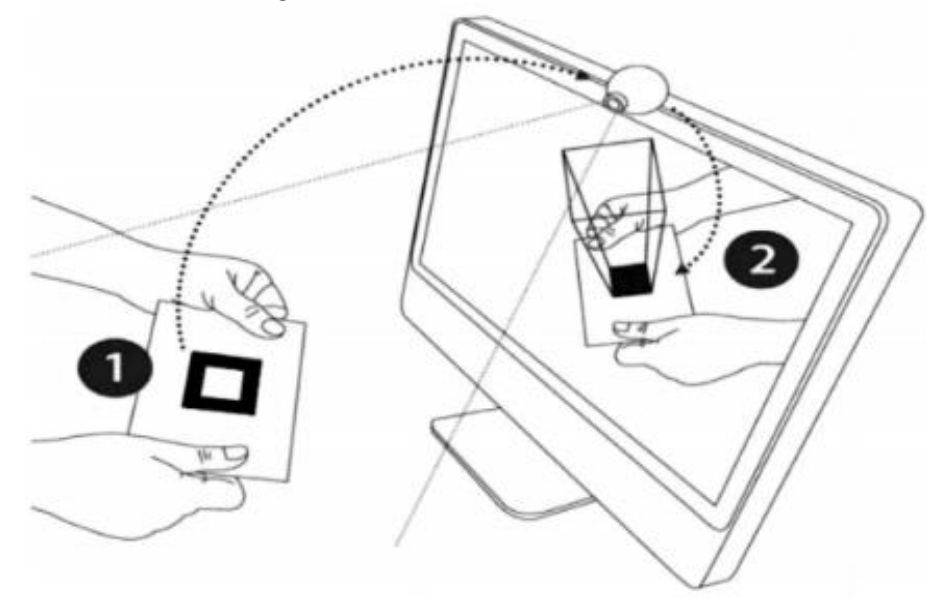

Fonte: Bazarov et. al (2017).

\section{RESULTADOS}

Algumas páginas utilizadas nas primeiras implementações, que demonstram a viabilidade do projeto, estão na Figura 4.

Figura 4 - Implementação inicial do aplicativo desenvolvido.
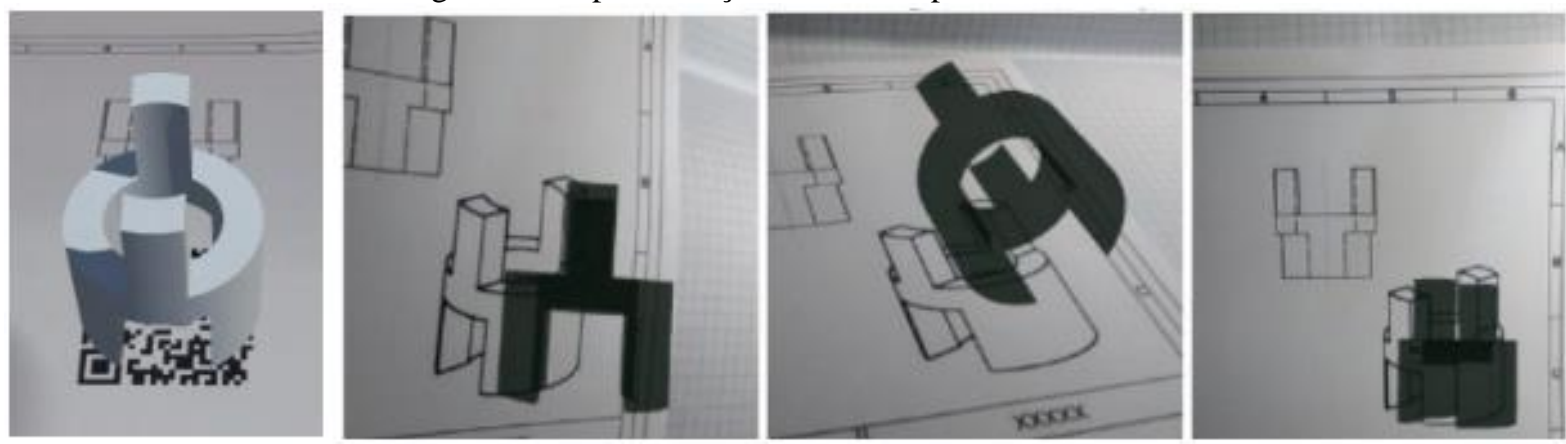

Fonte: Autores.

Trata-se de um exemplo da disciplina de desenho técnico mecânico com vistas ortogonais e perspectiva isométrica. Os alunos iniciantes nessa disciplina, muitas vezes, apresentam dificuldades ao relacionar as vistas com as perspectivas e vice-versa. Com o recurso de RA apresentado, pode-se interagir dinamicamente com as perspectivas, facilitando no processo de aprendizagem.

$\mathrm{Na}$ implementação inicial, diferentes tipos de imagem target com diferentes resoluções e tamanhos foram comparadas para que houvesse a leitura de forma adequada pela câmera dos dispositivos. É preferível que as imagens sejam com boa saturação em relação ao papel ou outra superfície que as exibirá. Estas comparações estão na Figura 5. 
Figura 5 - Imagens targets com $Q R$ Code e com uma imagem com saturação adequada.
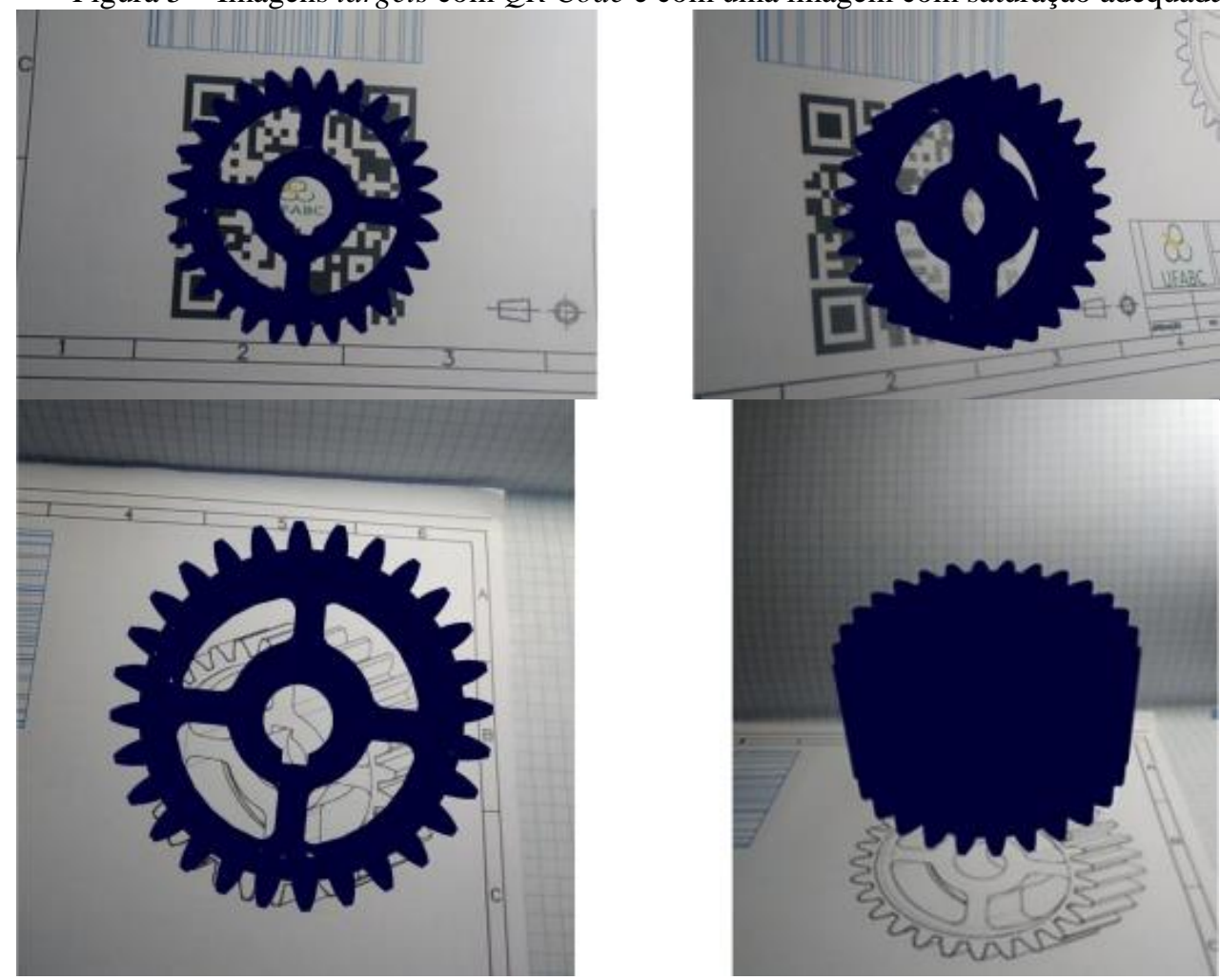

Fonte: Autores.

Na utilização da disciplina Elementos de Máquinas, a aplicação de RA foi implementada como na Figura 6.

Figura 6 - Implementação em exercício de Elementos de Máquinas.
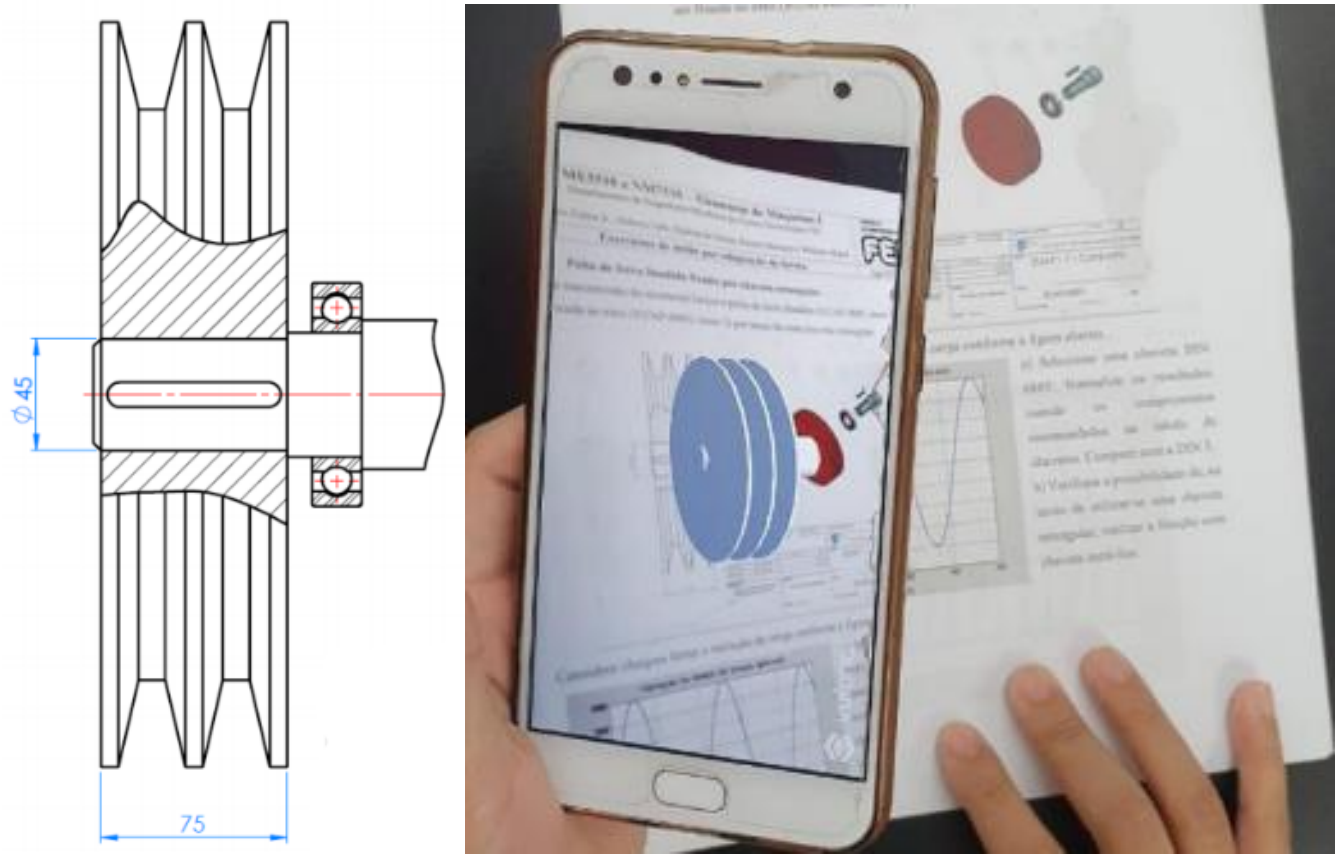

Fonte: Autores. 
Por meio de programação, é possível adicionar barra de ferramentas e implementar outros recursos para a interação com o modelo $3 \mathrm{D}$, como rotacioná-lo e modificar o material que é exibido, inserir vídeos, entre outros.

Esses fatos tornam a plataforma Unity associada a Vuforia adequadas para o desenvolvimento do aplicativo. Além disso, é possível criar um software inicial e convertê-lo para diferentes versões dos sistemas operacionais mais utilizados, como iOS e Android.

\section{CONCLUSÕES}

O trabalho de pesquisa demonstrou a aplicabilidade de RA na educação da engenharia tanto pelo estudo bibliográfico, quanto pelo desenvolvimento de um aplicativo, com tal finalidade. Embora aqui utilizado para ensino de desenho técnico, elementos de máquinas, pode-se concluir que também agregaria ao ensino de outras disciplinas em que a visualização tridimensional é desejada e contribui para o aprendizado.

A Plataforma Unity associada a Vuforia mostraram-se viáveis para o desenvolvimento do aplicativo, por ser possível implementar diferentes recursos ao aplicativo além de utilizá-lo simplesmente para a exibição de objetos do mundo virtual, como apresentação de vídeos, animações. O fato de ser um ambiente de desenvolvimento multiplataforma proporciona o acesso da aplicação a diferentes usuários, que nem sempre utilizam smartphones ou tablets com o mesmo sistema operacional.

Exemplos foram implementados para serem utilizados na disciplina de Fundamentos de Desenho Técnico, nos cursos de Engenharia da UFABC, com o livro utilizado em sala de aula. No Centro Universitário FEI, exemplos foram implementados na disciplina de Elementos de Máquinas no curso de Engenharia Mecânica.

Diferentes recursos complementares estão em análise para serem implementados no aplicativo, visto que a pesquisa de Iniciação Científica pela UFABC está em execução.

\section{REFERÊNCIAS}

AKÇAYIR, M. et al. Augmented reality in science laboratories: The effects of augmented reality on university students' laboratory skills and attitudes toward science laboratories. Computers in Human Behavior, [S. l.], v. 57, p. 334-342, 2016. Disponível em: https://doi.org/10.1016/j.chb.2015.12.054

ALIEV, Y. et al. 3D augmented reality software solution for mechanical engineering education. ACM International Conference Proceeding Series, [S. l.], v. Part F1320, p. 318-325, 2017. Disponível em: https://doi.org/10.1145/3134302.3134306

ANTONIOLI, M.; BLAKE, C.; SPARKS, K. Augmented Reality Applications in Education. The Journal of Technology Studies, [S. l.], v. XL number, p. 96-107, 2014. Disponível em: https://doi.org/https://doi.org/10.21061/jots.v40i2.a.4

AZUMA, R. T. A survey of augmented reality. In Presence: Teleoperators and Virtual Environments 6, 4, [S. l.], v. 6, 4, p. 355-385, 1997. Disponível em: https://doi.org/10.1561/1100000049

BACCA, J. et al. Augmented reality trends in education: A systematic review of research and applications. Educational Technology and Society, [S. l.], v. 17, n. 4, p. 133-149, 2014. 
BAZAROV, S. E. et al. Applying Augmented Reality in practical classes for engineering students. IOP Conference Series: Earth and Environmental Science, [S. l.], v. 87, n. 3 , 2017. Disponível em: https://doi.org/10.1088/1755-1315/87/3/032004

BILLINGHURST, M.; CLARK, A.; LEE, G. A survey of augmented reality. Foundations and Trends in Human-Computer Interaction, [S. l.], v. 8, n. 2-3, p. 73-272, 2014.

Disponível em: https://doi.org/10.1561/1100000049

CAUDELL, T. P.; MIZELL, D. W. Augmented reality: an application of heads-up display technology to manual manufacturing processes. Twenty-Fifth Hawaii International Conference on System Sciences. Kauai, HI, USA: IEEE, 1992. p. 659-669. Disponível em: https://doi.org/10.1109/hicss.1992.183317

DIEGMANN, P. et al. Benefits of Augmented Reality in Educational Environments - A Systematic Literature Review. 12th International Conference on Wirtschaftsinformatik, March 4-6 2015, Osnabrück, Germany, [S. l.], n. March, p. 1542-1556, 2015.

FIALHO, A. B. Realidade Virtual E Aumentada - Tecnologias Para Aplicações

Profissionais. São Paulo: Editora Érica, Saraiva, 2018. E-book.

GARZÓN, J.; ACEVEDO, J. Meta-analysis of the impact of Augmented Reality on students' learning gains. Educational Research Review, [S. l.], v. 27, n. April, p. 244-260, 2019. Disponível em: https://doi.org/10.1016/j.edurev.2019.04.001

GARZÓN, J.; PAVÓN, J.; BALDIRIS, S. Systematic review and meta-analysis of augmented reality in educational settings. Virtual Reality, [S. l.], v. 23, n. 4, p. 447-459, 2019.

Disponível em: https://doi.org/10.1007/s10055-019-00379-9

IBÁÑEZ, M. B.; DELGADO-KLOOS, C. Augmented reality for STEM learning: A systematic review. Computers and Education, [S. l.], v. 123, n. April, p. 109-123, 2018. Disponível em: https://doi.org/10.1016/j.compedu.2018.05.002

INSTITUTO BRASILEIRO DE GEOGRAFIA E ESTATÍSTICA. PNAD 2017: Acesso à Internet e à Televisão e Posse de Telefone Móvel Celular Para Uso Pessoal. Pesquisa Nacional por Amostra de Domicílios, [S. l.], p. 1-12, 2018. Disponível em: https://biblioteca.ibge.gov.br/visualizacao/livros/liv101631_informativo.pdf

MARTIN, S. et al. New technology trends in education: Seven years of forecasts and convergence. Computers and Education, [S. l.], v. 57, n. 3, p. 1893-1906, 2011. Disponível em: https://doi.org/10.1016/j.compedu.2011.04.003

MEKNI, M.; LEMIEUX, A. Augmented Reality : Applications, Challenges and Future Trends. Applied Computational Science anywhere, [S. l.], p. 205-214, 2014.

OZDEMIR, M. et al. The Effect of Augmented Reality Applications in Learning Process: A Meta-Analysis Study. Egitim Arastirmalari - Eurasian Journal of Educational Research, 
[S. l.], v. 2018, n. 74, p. 165-186, 2018. Disponível em: https://doi.org/10.14689/ejer.2018.74.9

PTC VUFORIA. Augmented Reality. [s. l.], [s. d.]. Disponível em: https://www.ptc.com/en/products/augmentedreality. Acesso em: 29 jul. 2019.

RADU, I. Augmented reality in education: A meta-review and cross-media analysis. Personal and Ubiquitous Computing, [S. l.], v. 18, n. 6, p. 1533-1543, 2014. Disponível em: https://doi.org/10.1007/s00779-013-0747-y

RIASCOS, L. A. M. et al. Fundamentos de Desenho Técnico. v. 1 ed. São Paulo: Editora Plêiade, 2018. E-book.

SANTOS, M. E. C. et al. Augmented reality learning experiences: Survey of prototype design and evaluation. IEEE Transactions on Learning Technologies, [S. l.], v. 7, n. 1, p. 38-56, 2014. Disponível em: https://doi.org/10.1109/TLT.2013.37

UNITY. Unity User Manual (2019.1). [s. l.], [s. d.]. Disponível em: https://docs.unity3d.com/Manual/index.html. Acesso em: 29 jul. 2019.

YIM, M. Y. C.; CHU, S. C.; SAUER, P. L. Is Augmented Reality Technology an Effective Tool for E-commerce? An Interactivity and Vividness Perspective. Journal of Interactive Marketing, [S. l.], v. 39, p. 89-103, 2017. Disponível em:

https://doi.org/10.1016/j.intmar.2017.04.001

\title{
DEVELOPMENT OF AN AUGMENTED REALITY APPLICATION FOR TEACHING OF TECHNICAL DESIGN, MACHINE ELEMENTS AND MECHANICAL DESIGN
}

\begin{abstract}
Augmented Reality $(A R)$ is an interactive technology that introduces virtual objects or images into the real world. This technology can be used in applications for smartphones and tablets, without a computer with high processing power. AR is a suitable technology to be applied in the educational field. The user interact with the object of study, stimulating their interest and learning. This article presents the development of an application compatible with different operational systems for smartphones, to assist teaching technical design, machine elements and mechanical projects. The application can be used at different moments for learning: either in pre-preparation for classes, during classes or after them, to review the contents studied or to perform exercises. This project is a partnership between Federal University of $A B C(U F A B C)$ and Centro Universitário FEI. In each institution, two scientific initiation projects were approved and are under development and tests.
\end{abstract}

Keywords: Augmented Reality. Education. Engineering. Technical Design. Mechanical Design. 